\title{
Modelling and Parameters Study of Piezoceramic Parts of an Electroacoustic Transducers
}

\author{
Nowrouz M. Nouri, Mohammad Riahi and Hamid R. Gharavian \\ Department of Mechanical Engineering, Iran University of Science and Technology, Narmak, Tehran, Iran, \\ 1684613114.
}

Ali Valipour
Department of Engineering, Chamran University of Ahvaz, Ahvaz, Iran, 6135783151.

(Received 18 December 2013; accepted 16 February 2015)

Electroacoustic transducers as transmitters and receivers play major role in underwater communication systems. Piezoceramic rings are the most important parts of electroacoustic transducers. In this investigation, attempts were made to use the matrix model and the finite element model to evaluate frequency behaviour of piezoceramic rings. In order to validate the accuracy of the proposed models and the solution algorithm, results obtained from both models were compared with experimental results presented by Radmanović, et al. Upon confirmation of the obtained results from the two models, the effects of the geometrical parameters on the frequency response of the ring and the surrounding domain in which the ring oscillates were studied. Based on the obtained results, the geometrical parameters have effects on both the frequency resonance of the ring, as well as the value of the electrical impedance. It is also noted that the surrounding domain only causes change on the intensity of the ring's electrical impedance.

\section{INTRODUCTION}

The use of acoustic waves for the recognition and location identification of underwater bodies is called sonar. ${ }^{1-3}$ Compared to the other form of wave transmission, acoustic waves are more suitable for underwater applications due to low attenuation. ${ }^{3-5}$ Consequently, underwater applications of sonar are related to acoustic fundamentals. Due to military needs, especially during world wars, the related technologies associated with sonar have developed. ${ }^{6}$ A transducer is a device capable of converting one form of energy into another. ${ }^{7}$ The underwater transducers convert electric energy into a sound wave, which is mechanical energy, and vice versa. ${ }^{6,8}$ Careful design and modelling of transducers is necessary, since it is crucial that they perform at the desired level. Moreover, recognition of the frequency behaviour of piezoceramic rings has high importance, since they are the main parts of transducers. The frequency behaviour of a piezoceramic ring is usually obtained by studying its impedance curve and calculating its resonance frequencies. ${ }^{9}$

Traditionally, the design of transducers and the study of the behaviour of their parts, particularly piezoceramic rings, has been done by using lumped parameters models. These models were made on the principle that by assuming one dimension, their different parts could be modelled as a group of masses connected by several springs and dampers. ${ }^{6,10}$ Since it is possible to equate mechanical parts with the forming elements of an electric circuit, the next step in modelling is to use equivalent circuits. Through using these circuits, the desired parameters for performance analysis, such as frequency resonance, bandwidth, etc., would be obtained. ${ }^{11}$ Another analytical model that can be used is the 2D matrix model. In this model, the re- lations of forces acting on surfaces, different surface velocities, voltage, and current of a piece like a piezoceramic ring would be expressed in the form of a matrix relation and an impedance matrix of $5 * 5$. This model is also capable of considering the effects of the acoustic environment. For parts like metal rings not containing voltage and current, the size of impedance matrix would be reduced to $4 * 4{ }^{12,13}$ Since it was not possible to use analytical models to analyse transducers with complex geometries, finite element methods have been used for the design and analysis of these transducers. So, the main advantages of finite element analysis is the possibility of precise and complete modelling of all parts with complex geometries. ${ }^{14,15}$

In light of the points discussed, the present study utilizes matrix models as well as finite element models, and applies them to simulate a piezoceramic ring. These models can be considered advanced methods in the modelling of the electroacoustic transducers parts. In order to validate the used algorithm, initially for a specified piezoceramic ring, the obtained frequency response was compared to valid results presented by experimental studies in credible references. Then, the frequency response of a piezoceramic ring for several dimensional conditions and different environments were studied. Finally, by interpreting these results through analytical models, fundamentals of vibrations, and circuit analysis, the effects of different parameters on the considered frequency response was evaluated.

\section{METHODS OF MODELLING PIEZOCERAMIC RINGS}

The design and modelling of underwater transducers is possible through the recognition of piezoceramic rings' behaviour 


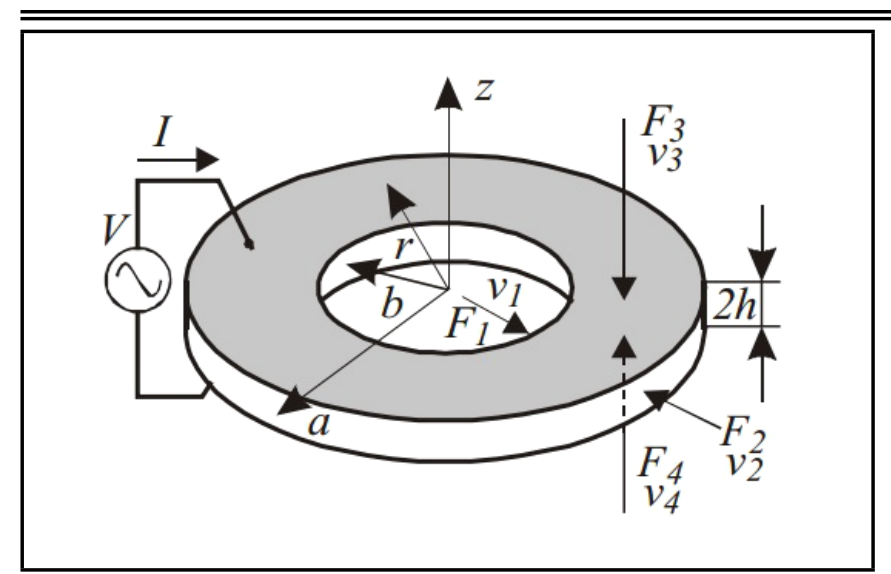

Figure 1. A piezoceramic ring (conventional directions of forces and velocities are shown).

as a major element in these transducers. Moreover, the modelling of a ring with the aim of reducing and improving production costs is rather important. The methods used in this study for the modelling of piezoceramic elements of underwater electroacoustic transducers will be described in this section.

\subsection{Matrix Model}

The matrix model is known as the most advanced analytical tool for the design and analysis of different elements used in the electroacoustic transducers. In order to better introduce the matrix model, its application in the analysis of a piezoceramic ring is presented. Figure 1 is a schematic view of a piezoceramic ring. This ring has stimulating voltage at its inlet and outlet.

$F_{i}$ are applied forces on the ring surfaces, and $v_{i}$ are surface velocities along different directions. By using stress-strain, displacement-strain relations and other related points, the following relations would be presented for impedance of a piezoceramic ring: ${ }^{16}$

$$
\begin{gathered}
{\left[\begin{array}{c}
F_{10} \\
F_{20} \\
F_{30} \\
F_{40} \\
V_{0}
\end{array}\right]=\left[\begin{array}{lllll}
z_{11} & z_{12} & z_{13} & z_{13} & z_{15} \\
z_{21} & z_{22} & z_{23} & z_{23} & z_{25} \\
z_{13} & z_{23} & z_{33} & z_{34} & z_{35} \\
z_{13} & z_{23} & z_{34} & z_{33} & z_{35} \\
z_{15} & z_{25} & z_{35} & z_{35} & z_{55}
\end{array}\right]\left[\begin{array}{c}
v_{10} \\
v_{20} \\
v_{30} \\
v_{40} \\
I_{0}
\end{array}\right] ;} \\
V=F_{i 0} e^{j \omega t} \\
V=V_{0} e^{j \omega t}
\end{gathered}
$$

In the above relations, $z_{i j}$ is a function of geometrical characteristics and physical structure parameters, as well as the frequency. ${ }^{6,12} F_{i 0}$ and $V_{0}$ are the force spectrum and fluctuating voltage. A matrix comprised of $z_{i j}$ is called the impedance matrix of a piezoceramic ring. In order to obtain the resonance frequencies of this ring, it suffices to equate the determinant of the matrix to zero. $\omega$ is the angular frequency, and it equates to

$$
\omega=2 \pi f \text {. }
$$

In the above relation, $f$ is the frequency in terms of hertz $(\mathrm{Hz})$. More important than defining the matrix impedance is defining the input electrical impedance. The input electrical impedance is the relation of voltage to current in the inlet electrical terminals. The input electrical impedance contains mechanical properties as well as acoustic loading. By drawing this impedance as a function of the frequency, it becomes possible to observe and calculate the resonance frequencies (minimum points) and anti-resonance frequencies (the maximum points). ${ }^{15}$ To draw this impedance, first it is necessary to express the relation between force and velocity at the external surfaces by using the acoustic impedance: ${ }^{12}$

$$
F_{i}=-Z_{i} v_{i}, \quad i=1,2,3,4 .
$$

Acoustic impedance is the product of density multiplied by velocity of sound in any acoustic domain. The amount of acoustic impedance for air is about 400, and for water is about $1.5^{*} 10^{6}$. A unit of acoustic impedance (external impedance) is $\left(\frac{\mathrm{kg}}{\mathrm{m}^{2} \mathrm{~s}}\right)$ or $\left(\frac{\mathrm{Pas}}{\mathrm{m}}\right)$, while a unit of obtained $z_{i j}$ impedance of in the matrix impedance is $\left(\frac{\mathrm{kg}}{\mathrm{s}}\right)$. It is evident that in order to unify the acoustic impedance with $z_{i j}$, acoustic impedance would have to be multiplied by a unit of surface. This surface is equal to the area that impedance enters on it. This means:

$$
\begin{aligned}
& Z_{1}=Z_{1_{-} \text {acousic }} \times 2 * \pi b * 2 h ; \\
& Z_{2}=Z_{2_{-} \text {acousic }} \times 2 * \pi a * 2 h ; \\
& Z_{3}=Z_{3_{-} \text {acousic }} \times \pi\left(a^{2}-b^{2}\right) ; \\
& Z_{4}=Z_{4_{-} \text {acousic }} \times \pi\left(a^{2}-b^{2}\right) .
\end{aligned}
$$

In order to obtain the input electrical impedance as a function of frequency, it is necessary to make some changes to Eq. (1):

$$
\begin{aligned}
& {\left[\begin{array}{c}
F_{10} \\
F_{20} \\
F_{30} \\
F_{40} \\
V_{0}
\end{array}\right]=\left[\begin{array}{lllll}
z_{11} & z_{12} & z_{13} & z_{13} & z_{15} \\
z_{21} & z_{22} & z_{23} & z_{23} & z_{25} \\
z_{13} & z_{23} & z_{33} & z_{34} & z_{35} \\
z_{13} & z_{23} & z_{34} & z_{33} & z_{35} \\
z_{15} & z_{25} & z_{35} & z_{35} & z_{55}
\end{array}\right]\left[\begin{array}{c}
v_{10} \\
v_{20} \\
v_{30} \\
v_{40} \\
I_{0}
\end{array}\right] \stackrel{F_{i}=-Z_{i} v_{i}}{\longrightarrow}} \\
& {\left[\begin{array}{c}
-Z_{1} v_{10} \\
-Z_{2} v_{20} \\
-Z_{3} v_{30} \\
-Z_{4} v_{40} \\
V_{0}
\end{array}\right]=\left[\begin{array}{lllll}
z_{11} & z_{12} & z_{13} & z_{13} & z_{15} \\
z_{21} & z_{22} & z_{23} & z_{23} & z_{25} \\
z_{13} & z_{23} & z_{33} & z_{34} & z_{35} \\
z_{13} & z_{23} & z_{34} & z_{33} & z_{35} \\
z_{15} & z_{25} & z_{35} & z_{35} & z_{55}
\end{array}\right]\left[\begin{array}{c}
v_{10} \\
v_{20} \\
v_{30} \\
v_{40} \\
I_{0}
\end{array}\right] ; \quad(7)}
\end{aligned}
$$

$\left[\begin{array}{c}0 \\ 0 \\ 0 \\ 0 \\ V_{0} / I_{0}\end{array}\right]=$

$$
\left[\begin{array}{ccccc}
z_{11}+Z_{1} & z_{12} & z_{13} & z_{13} & z_{15} \\
z_{21} & z_{22}+Z_{2} & z_{23} & z_{23} & z_{25} \\
z_{13} & z_{23} & z_{33}+Z_{3} & z_{34} & z_{35} \\
z_{13} & z_{23} & z_{34} & z_{33}+Z_{4} & z_{35} \\
z_{15} & z_{25} & z_{35} & z_{35} & z_{55}
\end{array}\right]\left[\begin{array}{c}
v_{10} / I_{0} \\
v_{20} / I_{0} \\
v_{30} / I_{0} \\
v_{40} / I_{0} \\
1
\end{array}\right]
$$

By considering the first four lines of the above matrix and the point that $z_{i j}$ is determined, it becomes possible to find unknowns terms $\left\{\left(v_{10} / I_{0}\right),\left(v_{20} / I_{0}\right),\left(v_{30} / I_{0}\right)\right.$, and $\left.\left(v_{40} / I_{0}\right)\right\}$ in any specific frequency. Then, by using the following equation, which is related to the fifth line of the above matrix, it becomes possible to find the input electrical impedance for a piezoceramic ring:

$$
z_{\mathrm{ul}}=V_{0} / I_{0}=z_{15} \frac{v_{10}}{I_{0}^{2}}+z_{25} \frac{v_{20}}{I_{0}^{2}}+z_{35} \frac{v_{30}}{I_{0}^{2}}+z_{35} \frac{v_{40}}{I_{0}^{2}}+\frac{z_{55}}{I_{0}^{2}} .
$$


In the above equation, $z_{\mathrm{ul}}$ is the input electrical impedance. It is evident that in order to calculate $z_{\mathrm{ul}}$, all acoustic impedances would have to be known. ${ }^{12}$ By drawing the related curve of the above equation, it becomes possible to analyse the behaviour of the piezoceramic ring caused by the applied voltage.

In addition to the high precision in the matrix method, attention needs to be paid to the fact that in utilizing this method, there are two major limitations. First, by making the geometrical shape more complex, using this method and formation of the impedance matrix becomes more difficult. Second, even in simple geometrical shapes, when a large number of elements are present along each other in a transducer, making correct relationships between impedances of these elements and simultaneously analysing them would be very cumbersome. Therefore, in the present study, an attempt was made to develop a finite element method suitable for both modelling of complex geometrical shapes and for modelling transducers with many elements.

\subsection{Finite Element Method}

In recent years, due to necessity of using modelling of different and complex geometries, the utilization of finite element methods for analysis of electroacoustic transducers elements has increased. ${ }^{2}$ Acoustic analysis in finite elements could take place in both frequency domain and time domain. Of course, for this type of problem, frequency domain is usually used. The major task in the finite element method modelling is the correct and precise defining of the model's boundary conditions. Initially, the propagation domain around the transducer surface could be modelled. In cases where vibrating bodies are axisymmetric, it would be better to use axisymmetric models. This helps to reduce the computational time. Introducing the materials and properties of different parts and domains, as well as determining the base voltage for piezoelectric materials, is the next step in the definition of the problem. ${ }^{17,18}$ Figure 2 shows finite element modelling of a single ring along with its acoustic domain.

In Fig. 2, the upper part shows the ring along with its considered spherical acoustic domain. In the lower part, the magnified schematic of ring is shown. The size of the acoustic domain around the vibrating surface of the ring has been made much larger than ring's dimensions. This was done to model infinite domain around the ring. Also, this modelling causes propagated waves to have the chance for receiving the effects of the domain and related boundary conditions. ${ }^{14}$ After modelling, the ring and the domain must be meshed. An important point in the size selection of each element is to consider that in acoustical analysis, the size of the largest element must be a function of sound velocity and the highest performing frequency. Therefore, the size of the largest element could be calculated from the following relation:

$$
\text { Maximum element size } \leq \frac{c}{n \cdot f_{\max }} .
$$

In the above equation, $c$ is the velocity of sound in the acoustic domain, $f_{\max }$ is the highest frequency within the analysed frequency spectrum, and $n$ is a number between 8 and $12 .{ }^{19}$

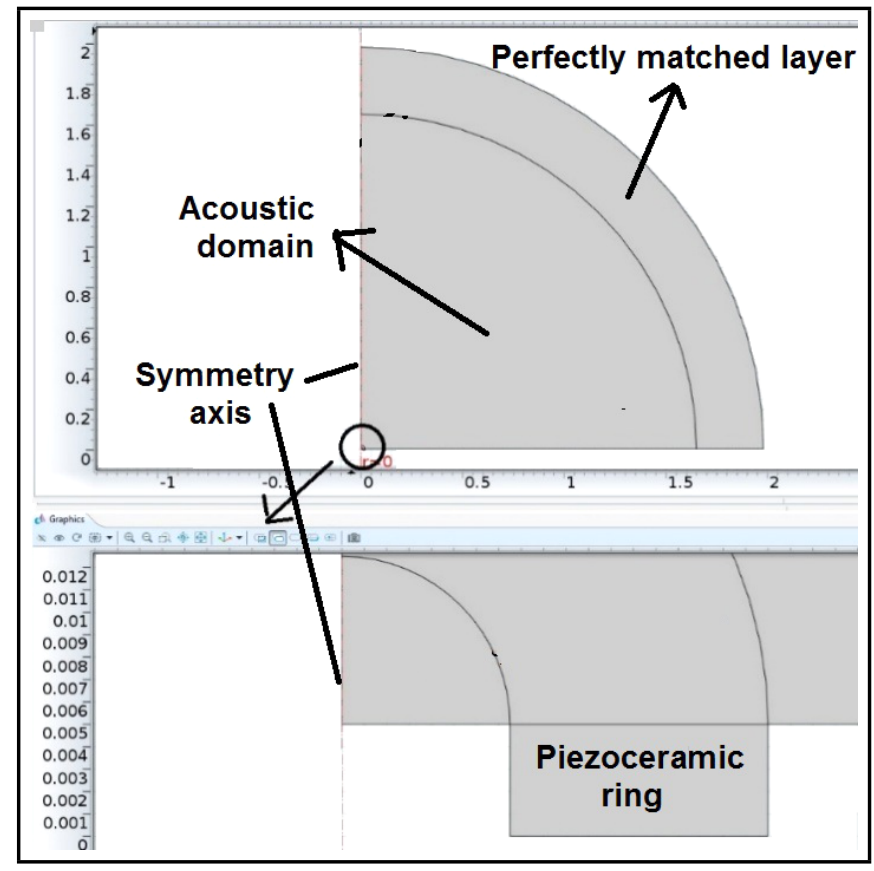

Figure 2. Schematic of acoustical calculation environment model (upper) and piezoceramic ring (lower).

\section{VALIDATION}

The piezoceramic ring in the simulations has an external diameter equal to $38 \mathrm{~mm}$, an internal diameter of $15 \mathrm{~mm}$, and a thickness $5 \mathrm{~mm}$. The ring's material is PZT8. These dimensions were selected by considering the sample ring tested in the work of Radmanović and Mančić. ${ }^{16}$

It was assumed that the ring passes a 1-volt simulation electrically. In continuation, the frequency analysis of this ring was done through different methods, and the results were compared. Also, in order to verify the obtained results from both methods, these results were compared to the experimental results presented by Radmanović and Mančić. ${ }^{16}$ In the matrix model, the first impedance matrix of the considered ring was obtained as a function of frequency. Then, by drawing the matrix for different values of frequency, the curve of the ring impedance was obtained. As mentioned earlier, the effects of the acoustic environment around the ring were entered into the model as acoustic impedance. Also, in the finite element method, after the modelling of the ring and the computational acoustic domain, the behaviour of the ring in the frequency domain was studied. Figure 3 depicts the obtained results from the matrix model and the finite element model next to each other.

The curve in Fig. 3 has many maximum and minimum points. Some of these maximum and minimum points could be representative of resonance and anti-resonance frequencies. As expected, a piezoceramic ring being freely vibrated has several main resonance modes, while other resonance modes like torsional modes, etc., could also be observed. Considering that the matrix model could only model motion along the radius and thickness, only major resonance modes would be observable by it. The finite element model is also two dimensional and axisymmetric. This means that only major resonance modes would be shown. Also, according to the figure, 


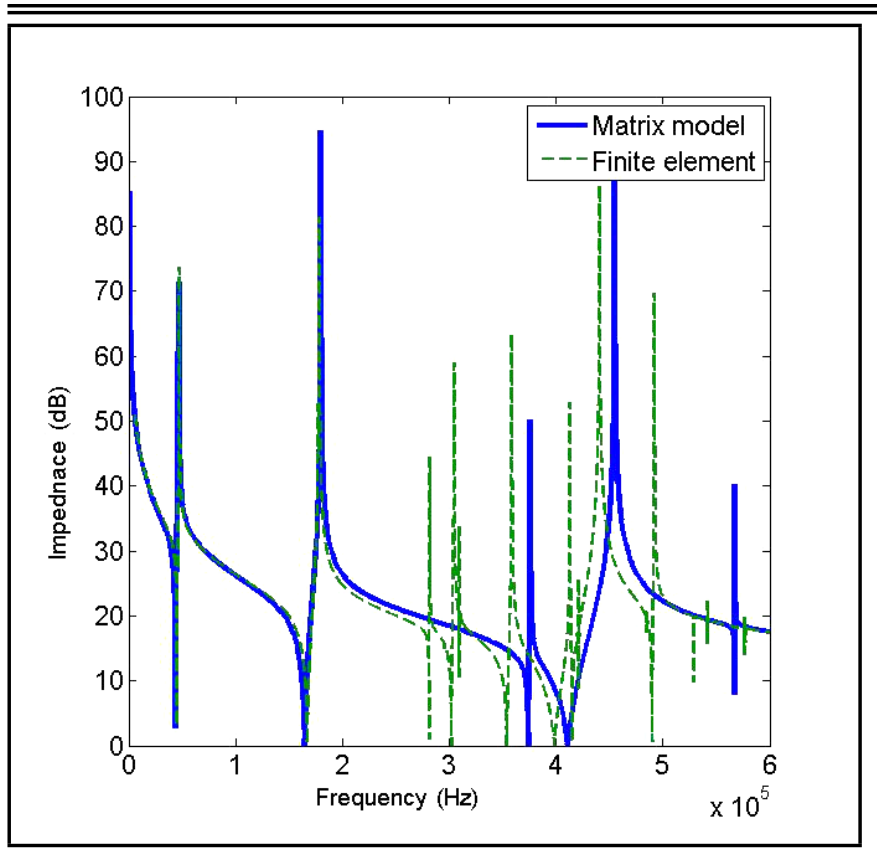

Figure 3. Comparison of the obtained results from the matrix model and the finite element model.

it is observed that the finite element model shows more resonance modes in a frequency spectrum when compared to the matrix model. The existence of these additional modes could be justified by parameters such as meshing. ${ }^{15}$ Thus, they could not be called major modes. It is worth mentioning that, in the above model, the environment surrounding the ring was completely assumed to be air. This means that all acoustic impedances were considered as air impedance:

$$
Z_{1}=Z_{2}=Z_{3}=Z_{4}=\rho_{\text {air }} \times c_{\text {air }}=400 \text { Rayl }
$$

Although the unit of impedance is ohm $(\Omega)$, it could be expressed in decibels $(\mathrm{dB})$ by using the following relation: ${ }^{16}$

$$
z_{\mathrm{ul}}=20 \log \left(\frac{Z_{\mathrm{ul}}}{50}+1\right) .
$$

In order to verify the results, they would have to be compared with experimental results. Consequently, the experimental results from the above mentioned ring in the work of Radmanović and Mančić ${ }^{16}$ is shown in Fig. 4.

Comparing Figs. 3 and 4 indicates that a suitable fit exists between the results obtained from modelling and these experimental results. In Fig. 4, four resonance modes are named. Three of them are labelled with the letter (R), referring to radius modes, and another one is labelled with the letter (T), related to thickness mode. The number next to each letter represents its number; e.g. $\mathrm{R}_{2}$ is related to the second resonance radius mode. ${ }^{16}$ Recognizing different types of resonance modes has high importance in studying frequency response. Therefore, it should be possible to recognize different modes for a known geometry. One common method to do this for a piezoceramic ring is to use frequency coefficients and utilize semiempirical simple relations. These relations could provide assistance in the recognition of different modes from a curve. For example, the main thickness mode, the first radius mode, and the second radius mode (circular) for the mentioned ring

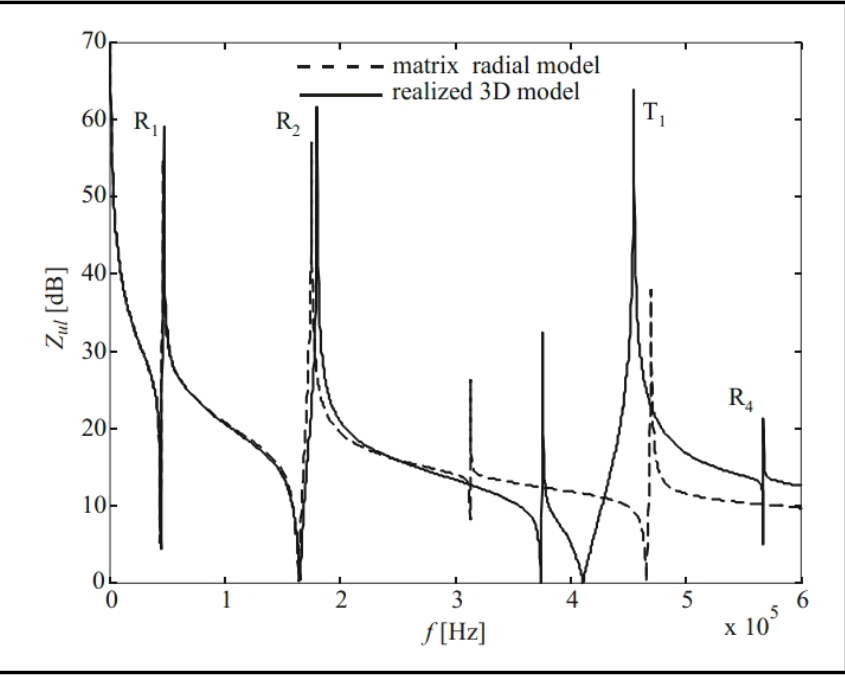

Figure 4. Comparison of obtained results from the matrix model and the experimental results in the work of Radmanović and Mančić. ${ }^{16}$

Table 1. Comparison of obtained results for the sample ring by different methods for modelling with experimental results.

\begin{tabular}{||l|ccc||}
\hline & $\begin{array}{c}\text { Radius } \\
\text { mode } \\
(\mathrm{kHz})\end{array}$ & $\begin{array}{c}\text { Hoop } \\
\text { mode } \\
(\mathrm{kHz})\end{array}$ & $\begin{array}{c}\text { Thickness } \\
\text { mode } \\
(\mathrm{kHz})\end{array}$ \\
\hline Experimental results & 46.0 & 168.0 & 418.0 \\
\hline Frequency constants method & 44.7 & 173.9 & 414.0 \\
\hline $\begin{array}{l}\text { Frequency constants method } \\
\text { relative error }(\%)\end{array}$ & 2.80 & 3.40 & 0.95 \\
\hline Matrix model & 43.2 & 179.0 & 417.3 \\
\hline Matrix model relative error $(\%)$ & 6.08 & 1.64 & 0.16 \\
\hline Finite element method & 43.0 & 178.0 & 418.2 \\
\hline $\begin{array}{l}\text { Finite element method relative } \\
\text { error }(\%)\end{array}$ & 2.19 & 6.50 & 0.04 \\
\hline
\end{tabular}

could be calculated by using the following relations: ${ }^{20}$

$$
\begin{aligned}
& f_{\text {thickness }}=\frac{N_{\text {thickness }}}{t}=\frac{2070 \mathrm{~Hz} \mathrm{~m}}{0.005 \mathrm{~m}}=414 \mathrm{kHz} \\
& f_{\text {radial }}=\frac{N_{\text {radial }}}{D}=\frac{1700 \mathrm{~Hz} \mathrm{~m}}{0.038 \mathrm{~m}}=44.7 \mathrm{kHz} \\
& f_{\text {hoop }}=\frac{N_{\text {hoop }}}{(D-d) / 2}=\frac{2000 \mathrm{~Hz} \mathrm{~m}}{(0.038 \mathrm{~m}-0.015 \mathrm{~m}) / 2}=173.9 \mathrm{kHz}
\end{aligned}
$$

In order to compare and validate the results quantitatively, the results from the semi-empirical matrix model and the finite element model have been compared to the experimental results in Table 1 .

It could be observed that with these semi-empirical relations, it is possible to estimate the main modes easily and precisely. The main advantage of the above calculations is to assist in recognizing different resonance modes. It is noteworthy that the terms $N$ in the above equations are frequency coefficients that could be found by referring to the available table for each standard piezoceramic ring. ${ }^{21}$ In Table 1, error values have been found by comparing the results of each model with the experimental results. Therefore, in light of Table 1, it

Table 2. Different conditions based on considered parameters.

\begin{tabular}{|c|cccc|}
\hline No. & $\begin{array}{c}\text { Acoustic } \\
\text { domain }\end{array}$ & $\begin{array}{c}\text { Thickness } \\
t_{c}(\mathrm{~mm})\end{array}$ & $\begin{array}{c}\text { Inner radius } \\
r_{\mathrm{ci}}(\mathrm{mm})\end{array}$ & $\begin{array}{c}\text { Outer radius } \\
r_{\mathrm{co}}(\mathrm{mm})\end{array}$ \\
\hline 1 & Air & 5 & 7.5 & $\{15: 5: 30\}$ \\
\hline 2 & Air & $\{5: 5: 20\}$ & 7.5 & 19 \\
\hline 3 & Water & 5 & 7.5 & 19 \\
\hline
\end{tabular}




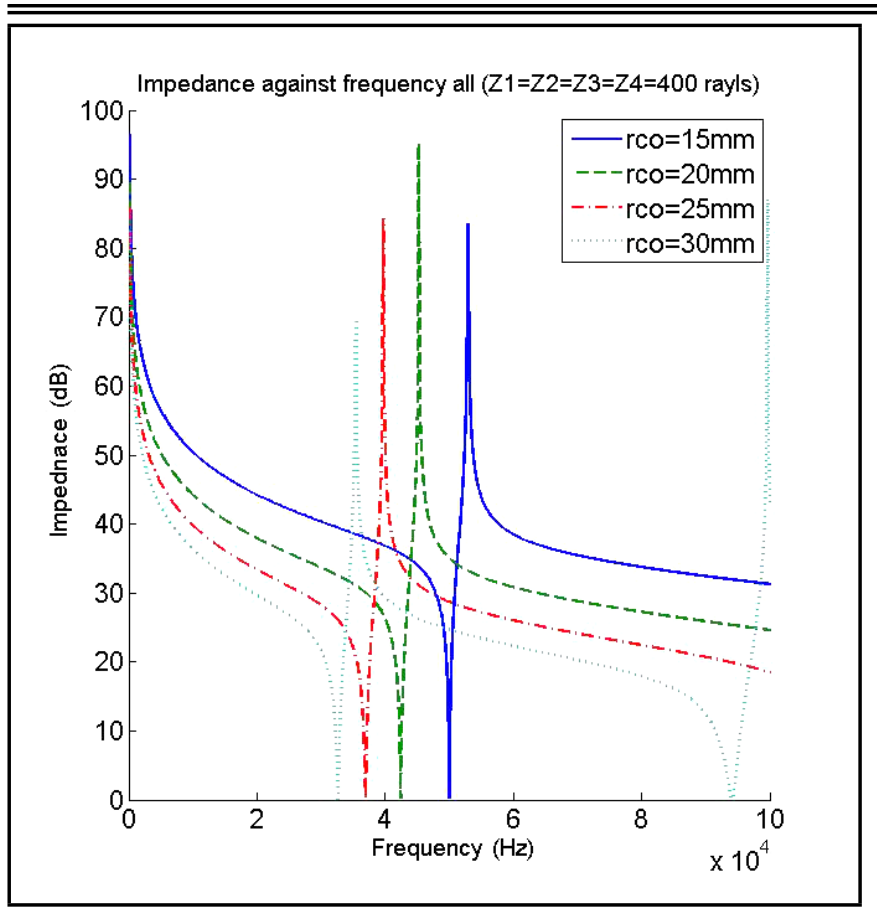

Figure 5. Survey of the effect of change in the external radius of the piezoceramic ring via the matrix model.

is safe to express that almost all methods present a good precision. Also, it is noticed that for the present problem with a simple geometry, in different modes, results obtained from the matrix models possess the best precision in comparison to other methods. It is obvious that where no difficulties would arise for complex geometries, this method could respond to the need.

\section{STUDY OF GEOMETRY AND ENVIRON- MENT PARAMETERS EFFECT ON FREQUENCY RESPONSE}

In proceeding and in order to determine effect of geometrical dimensions and acoustic domain of surrounding on different resonance modes of a ring, behaviour of the ring in a limited frequency range and in several different modes will be evaluated. Table 2 presents all studied conditions.

It is noteworthy that in row 1 , the external radius increases from $15 \mathrm{~mm}$ to $30 \mathrm{~mm}$ with iteration of $5 \mathrm{~mm}$ each. In the second row, the thickness also increases from $5 \mathrm{~mm}$ to $20 \mathrm{~mm}$, with $5 \mathrm{~mm}$ iterations. As depicted in the table, geometrical changes of the ring include changing in the external radius and its thickness. In order to observe the effect of the surrounding environment in the frequency response of the ring, the results for a certain geometry in two environments of air and water are compared to each other. The results obtained for each change in any of the three parameters, along with their interpretation, will be presented.

\section{RESULTS}

Obtaining the effect of different parameters in the frequency response of piezoceramic elements has high importance in the optimized and desired design of underwater transducers. In addition, by using a precise simulation of a ring, it would be

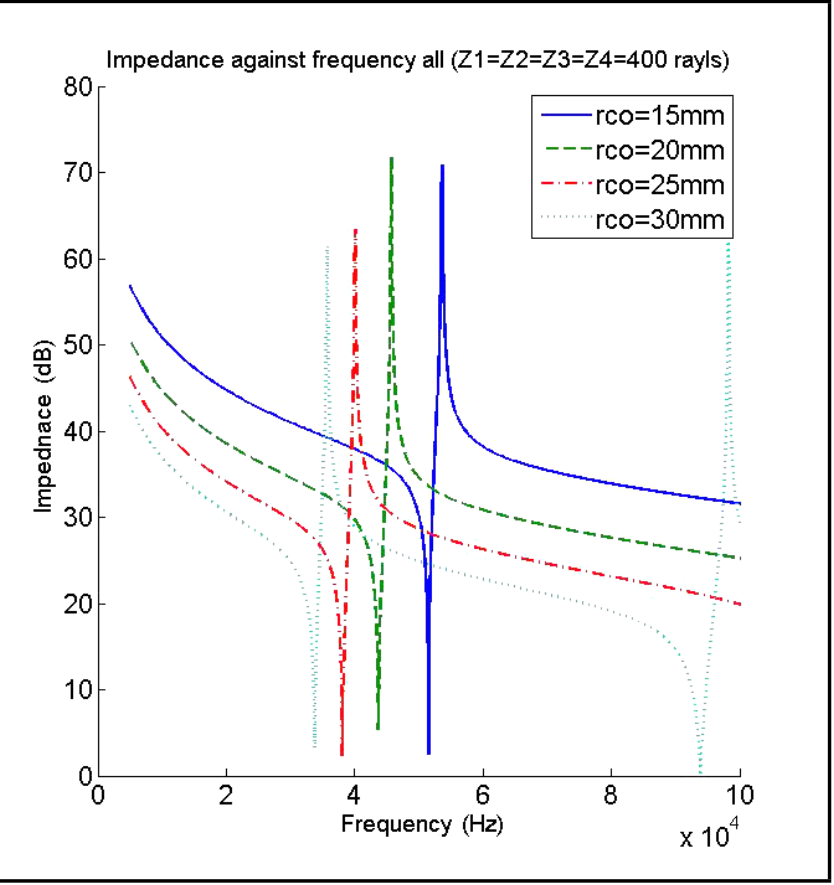

Figure 6. Survey of the effect of change in the external radius of the piezoceramic ring by using the finite element model.

possible to obtain the necessary knowledge in determining the effect of each parameter in the increase or decrease of the frequency resonance of a piezoceramic ring. In order to survey the effect of each introduced parameter in Table 2, the results obtained from the matrix model, as well as the finite element simulation, were gathered. These results were obtained in response to changes in three parameters: radius, thickness, and the surrounding environment. Through interpreting these results, it was possible to compare the effect value of different parameters.

\subsection{Condition 1}

In this condition, by assuming constant sizes of internal radii and piezoceramic ring thicknesses, the simulation takes place for four different sizes of the external radius. The selected frequency range is between 0 and $100 \mathrm{kHz}$. A diagram of the simulation results obtained from the matrix model is presented in Fig. 5, and the results obtained from the finite element model are presented in Fig. 6.

Figure 5 depicts the input electric impedance as a function for four rings with different external radii. This curve was drawn by using the matrix modelling method, and within a frequency range of 0 to $100 \mathrm{kHz}$. External radii of $15 \mathrm{~mm}$ to $30 \mathrm{~mm}$ were selected. For the external radius of $15 \mathrm{~mm}$, the first radial resonance frequency mode is approximately $48 \mathrm{kHz}$. By increasing the external radius and reaching $30 \mathrm{~mm}$, the resonance frequency decreased to $33 \mathrm{kHz}$. This means that with an increase of $100 \%$ in the radius, the resonance frequency decreased around $30 \%$. This is where the intensity of impedance in equal frequencies from the $15 \mathrm{~mm}$ radius to the $30 \mathrm{~mm}$ radius was decreased around $14 \mathrm{~dB}$. So, it could be stated that by increasing the external radius, the resonance frequency related to the radial mode decreased. Also, the level of input electrical impedance for a similar frequency decreased in 


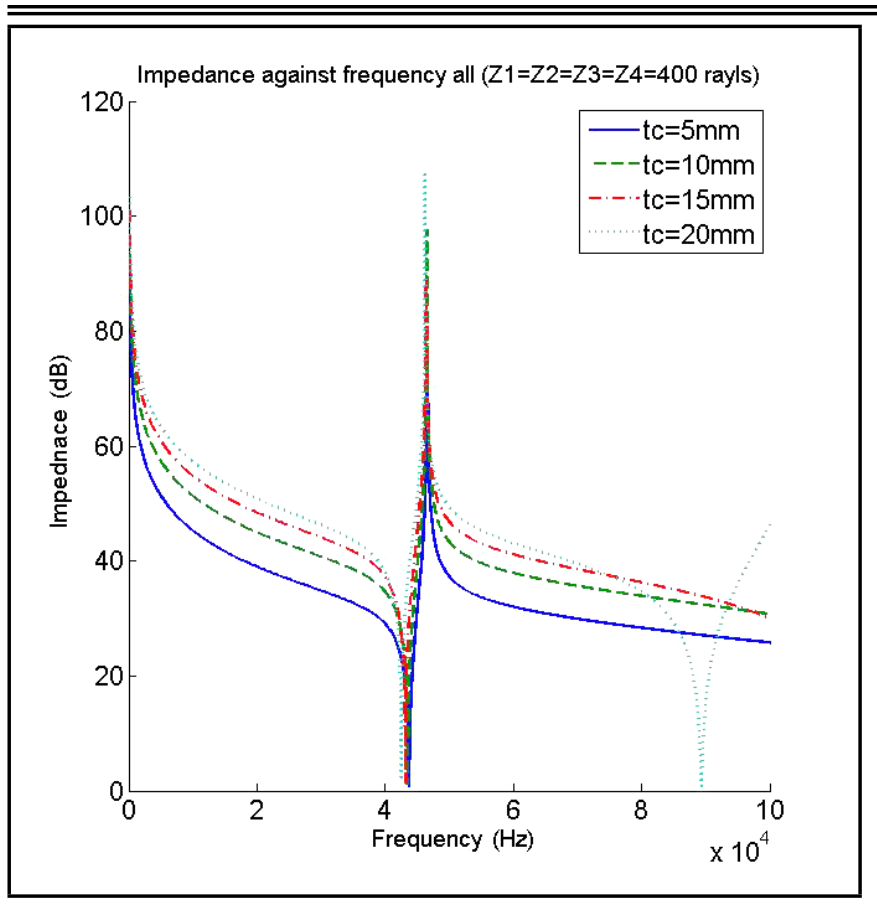

Figure 7. Survey of the effect of change in thickness of the piezoceramic ring through matrix modelling.

response to an increase in the external radius. Increasing the external radii translates to a change in the mass and stiffness of a piezoceramic ring. To study causes of this finding, sufficient information is needed about stiffness functions and mass, as well as frequency resonance and electrical impedance. More details about this point and the expression of needed relations will be introduced in the results interpretation section.

Figure 6 is similar to Fig. 5, except that it has been drawn by the finite element model. It is noticeable here that an increase in the external radius from $15 \mathrm{~mm}$ to $30 \mathrm{~mm}$ has caused the resonance frequency to reduce from $51 \mathrm{kHz}$ to about $35 \mathrm{kHz}$, meaning that by doubling the external radius, a reduction of $30 \%$ in the frequency resonance is noticeable. The reduction in the electric impedance value for this radius reduction is about $14 \mathrm{~dB}$. The objective of presenting this figure is to compare the results between the matrix modelling and the FEM modelling. It is noticeable that the results obtained from both models are in a very agreeable confirmation.

\subsection{Condition 2}

In this condition, by assuming fixed values for the internal and external radii, a simulation for four different sizes of thickness in a frequency range of 0 to $100 \mathrm{kHz}$ takes place. Figure 7 depicts a simulation by the matrix model on the basis of thickness.

In the above figure, the values of the input electrical impedance for four rings within different thicknesses are shown on the basis of frequency. This diagram was drawn through matrix modelling. It is noticeable that-contrary to the changes in the external radius-along with change in thickness, the frequency resonance related to the radius mode was not changed. This is where, similar to the condition of increase in the external radius with an increase in thickness, the level of the input electrical impedance for a known frequency goes

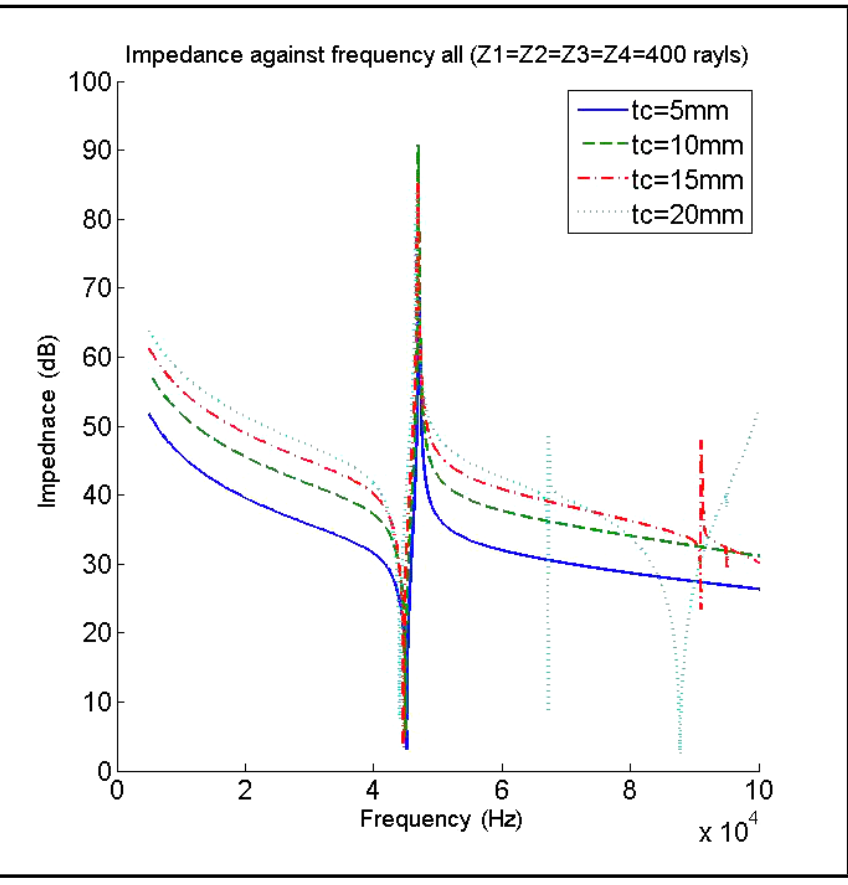

Figure 8. Survey of the effect of change in the piezoceramic ring thickness through FEM modelling.

up. Also, along with an increase in thickness, stiffness proportional to thickness decreases intensely. This leads to a reduction in the thickness frequency resonance. It is worth noting that the thickness frequency resonance for the designated ring is high (around $400 \mathrm{kHz}$ ), and in light of the selected frequency range, no thickness mode is observed. Similarly, in Fig. 8, also by utilizing the FEM, changes in ring thickness and its effect on the frequency response are shown.

A comparison between Figs. 7 and 8 reveals a good confirmation between the two methods of matrix and FEM modelling.

\subsection{Condition 3}

In this condition, by assuming fixed geometrical sizes, a simulation for two acoustical environments-water and airtakes place within a $300 \mathrm{kHz}$ to $500 \mathrm{kHz}$ range. The reason for selecting this frequency range is to be able to observe thickness modes as well as radial modes. Considering Fig. 1, the ring is connected to the surrounding domain from four sides. These four sides are radial sides, internal and external, and flat surfaces, upper and lower, along ( $z$ ) direction (thickness direction). In the first model, the ring is in touch with air from four sides. The second model is similar to the first one, with the difference that the upper flat surface (along the positive direction of $(z))$ is in contact with the water. Placing the ring in different environments causes different acoustic impedances on ring. The relation of acoustic impedance is as follows: ${ }^{2,16}$

$$
Z=\rho c .
$$

On the other hand, in the first model, the applied impedance on the different surfaces are:

$$
Z_{1}=Z_{2}=Z_{3}=Z_{4}=1 \frac{\mathrm{kg}}{\mathrm{m}^{3}} \times 400 \frac{\mathrm{m}}{\mathrm{s}}=400 \frac{\mathrm{Pa} \mathrm{s}}{\mathrm{m}} .
$$




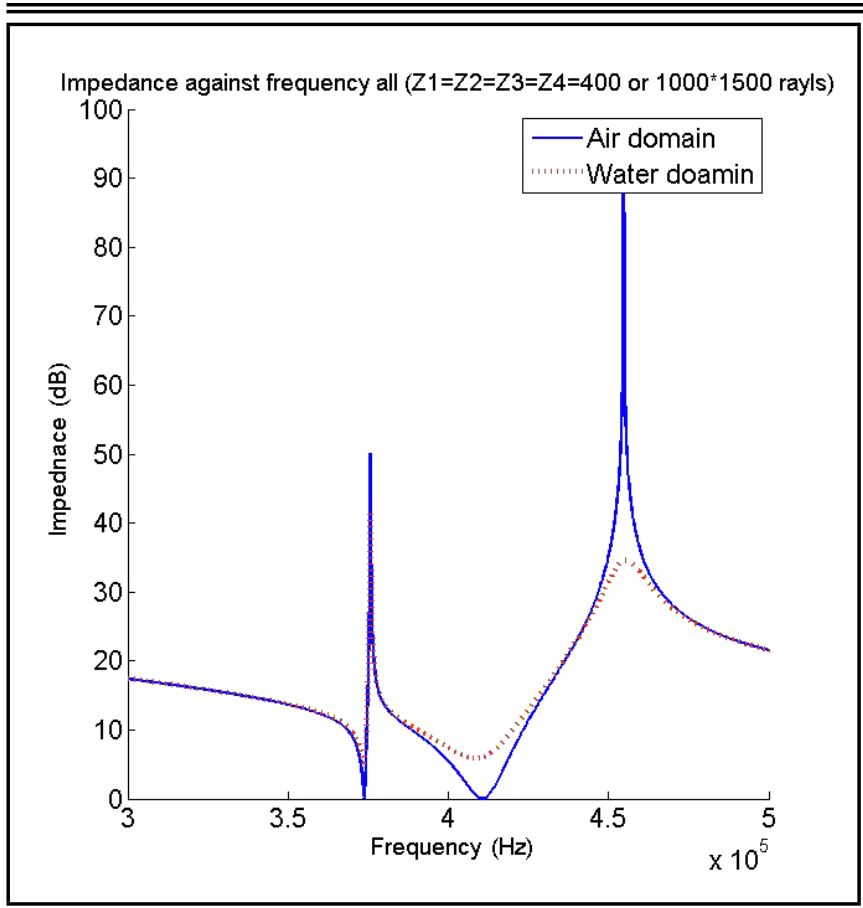

Figure 9. Survey of the effect of change in the acoustic environment material receiving propagation via the matrix model.

In the above equation, $z$ is the acoustic impedance, and indices 1 through 4, according to Fig. 1, are indicative of different directions of the ring. Also, the applied impedance on different surfaces in the second model will be as follows:

$$
\begin{aligned}
& Z_{1}=Z_{2}=Z_{3}=1 \frac{\mathrm{kg}}{\mathrm{m}^{3}} \times 400 \frac{\mathrm{m}}{\mathrm{s}}=400 \frac{\mathrm{Pa} \mathrm{s}}{\mathrm{m}} \\
& Z_{4}=1000 \frac{\mathrm{kg}}{\mathrm{m}^{3}} \times 1500 \frac{\mathrm{m}}{\mathrm{s}}=1.5 \times 10^{6} \frac{\mathrm{Pa} \mathrm{s}}{\mathrm{m}} .
\end{aligned}
$$

In Figs. 9 and 10, diagrams of electrical impedance of the ring are shown on the basis of the above two models. Figure 9 was obtained using the matrix model, and Fig. 10 was obtained by the FEM model.

According to Figs. 9 and 10, the blue line curve represents the condition in which the ring is in contact with air from four sides, whereas the red line curve represents the condition in which the ring is in contact with water from the upper side. The results are indicative that changes in the acoustic domain have little effect on the location of resonance frequencies. This is where changing the domain along its thickness from air to water has caused the impedance curve to become damped to a great extent. This means that no maximum or minimum points are vividly sharp. The difference between the results of the two models here is greater than in the previous conditions. This is because in higher frequencies, meshing becomes more important and has more effect on results. Perhaps this could mean that unsuitable meshing causes error in the obtained results. However, it is evident that the overall behaviour of results obtained from two models are similar to each other.

\section{RESULTS AND INTERPRETATIONS}

In order to analyse the effects of change in geometrical dimensions of a piezoceramic ring on impedance and resonance frequency, it is necessary to use analytical models. Any oscillating body in mechanics could be equated with a system

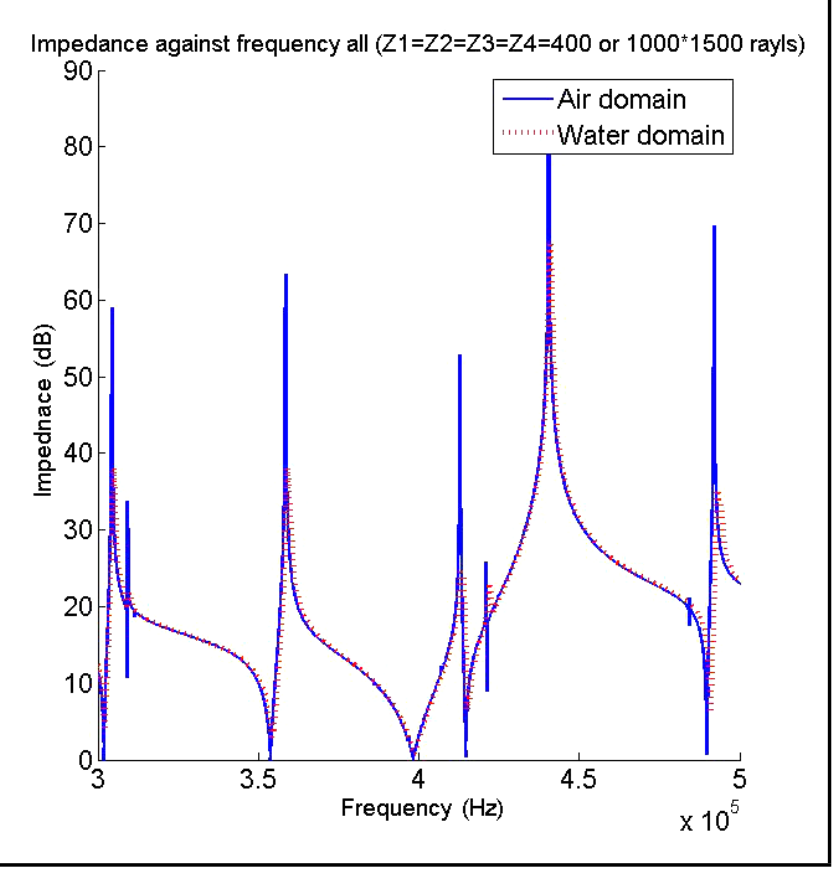

Figure 10. Survey of the effect of change in the material of acoustic environment receiving propagation via FEM model.

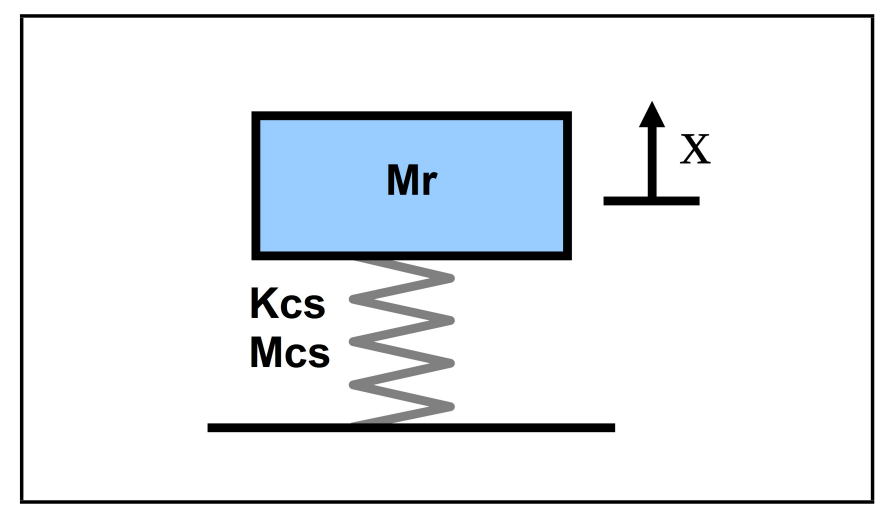

Figure 11. Systems of mass and spring equivalent to a vibrating piezoceramic ring.

of damper, mass, and spring. A piezoceramic ring could be equated with the mechanical system presented in Fig. 11.

Mass resulting from propagation in an acoustical environment is a function of acoustical domain properties, and the cross area of a projector as well as the frequency of propagation would be calculated from Eq. (14). In modelling for each system or structure, an equivalent mass has to be assumed. This mass could be considered as a combination of the mass of the ring $\left(M_{\mathrm{cs}}\right)$ and the added mass due to the propagation of the wave in the acoustic domain $\left(M_{\mathrm{r}}\right)$. It is noteworthy that $\left(M_{\mathrm{r}}\right)$ is usually small. Also for the ring, for every vibration direction, a mass with certain stiffness could be considered. The relation 


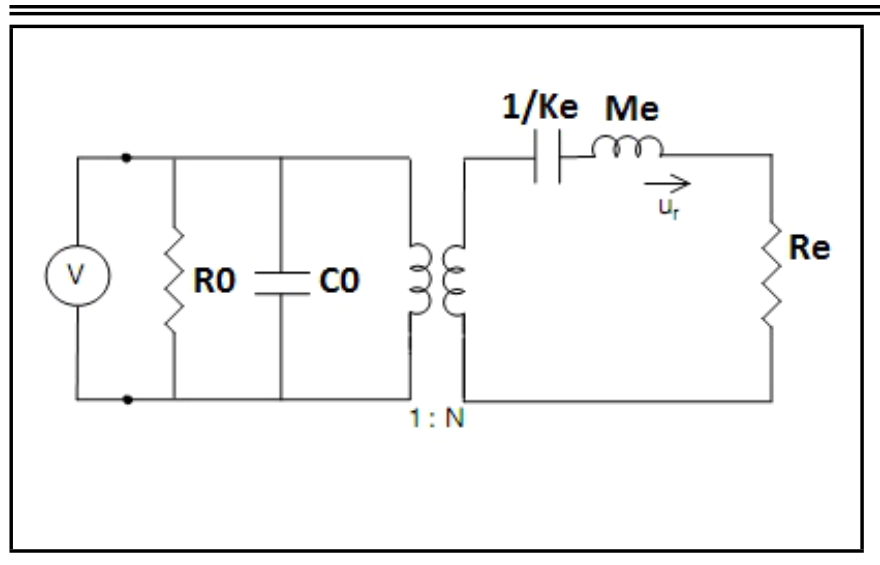

Figure 12. Equivalent electric circuit of a piezoceramic ring having stimulating voltage at both ends.

governing the mass and springs of Fig. 11 are as follows: ${ }^{10}$

$$
\begin{aligned}
& M_{\mathrm{r}}=\rho_{\mathrm{ac}} c_{\mathrm{ac}} A\left[\frac{2}{\pi}-J_{0}(k a)+\left(\frac{16}{\pi}-5\right) \frac{\sin (k a)}{k a}+\right. \\
& \left.\quad\left(12-\frac{36}{\pi}\right) \frac{1-\cos (k a)}{(k a)^{2}}\right] /(k a) ; \\
& M_{\mathrm{e}}=M_{\mathrm{r}}+\frac{M_{\mathrm{cs}}}{3} ; \\
& K_{\mathrm{e}}=\frac{A E}{l} .
\end{aligned}
$$

In the above relations, $\left(A_{\mathrm{c}}\right)$ is the cross area of circular portion of the ring's surface, $(E)$ is elasticity modules, and $(l)$ is ring's length along the vibration direction (thickness). $\left(\rho_{\mathrm{ac}}\right)$ is the density of the acoustic domain, $\left(c_{\mathrm{ac}}\right)$ is the sound velocity in the acoustic domain, $(a)$ is the radius of the projector cross area, $(A)$ is the cross area of the projector, $\left(J_{1}\right)$ is the first order Bessel function, and $k=2 \pi f / c_{\mathrm{ac}}$ is the wave number.

It was expressed earlier that, in all drawn impedance diagrams for the piezoceramic ring, the vertical axis is the input electrical impedance. The input electrical impedance for a ring stimulating voltage at its ends was obtained from Fig. 12 and based on Eqs. (16)-(22): ${ }^{19}$

$Z_{\mathrm{ul}}=\left[\frac{1}{R_{0}}+j \omega C_{0}+\frac{N^{2}}{Z_{\mathrm{mech}}}\right]^{-1}$

$R_{0}=\left(\omega C_{\mathrm{f}} \tan \delta\right)^{-1}$

$C_{0}=\left[\left(\frac{\varepsilon_{33}^{\mathrm{T}} A_{\mathrm{c}}}{t_{\mathrm{c}}}\right)\left(1-\frac{d_{33}^{2}}{\varepsilon_{33}^{\mathrm{T}} s_{33}^{\mathrm{E}}}\right)\right]$

$N=\frac{F}{V}=\frac{K_{\mathrm{c}} \Delta t_{\mathrm{c}}}{E t_{\mathrm{c}}}=\frac{\left(A_{\mathrm{c}} / t_{\mathrm{c}} s_{33}^{\mathrm{E}}\right)\left(S t_{\mathrm{c}}\right)}{E t_{\mathrm{c}}}=\frac{S}{E} \frac{A_{\mathrm{c}}}{t_{\mathrm{c}} s_{33}^{\mathrm{E}}}=\frac{d_{33} A_{\mathrm{c}}}{t_{\mathrm{c}} s_{33}^{\mathrm{E}}}$

$Z_{\mathrm{mech}}=\frac{K_{\mathrm{e}}}{j \omega}+j \omega M_{\mathrm{e}}+R_{\mathrm{e}}$

$R_{\mathrm{e}}=\rho_{\mathrm{ac}} c_{\mathrm{ac}} A\left(1-J_{1}(2 k a)\right) /(k a) ;$

$\omega_{n}=\sqrt{\frac{K_{\mathrm{e}}}{M_{\mathrm{e}}}}$.

The unit of the input electrical impedance obtained via Eq. (16) is ohm. By using Eq. (11), it could be calculated in
Table 3. Introduction of some parameters of piezoelectric materials.

\begin{tabular}{|lll|}
\hline Symbol & Definition & Unit \\
\hline$C_{\mathrm{f}}$ & Free capacitance & $\mathrm{C} / \mathrm{V}$ \\
$\tan \delta$ & Dielectric loss & 1 \\
$\varepsilon_{33}^{T}$ & Piezoelectric constant & $\mathrm{C} / \mathrm{mV}$ \\
$t_{\mathrm{c}}$ & Piezoceramic ring thickness & $\mathrm{m}$ \\
$A_{\mathrm{c}}$ & Piezoceramic ring cross area & $\mathrm{m}^{2}$ \\
$d_{33}$ & Piezoelectric strain constant & $\mathrm{C} / \mathrm{N}$ \\
$s_{33}^{\mathrm{E}}$ & Compliance matrix member & $1 / \mathrm{Pa}$ \\
\hline
\end{tabular}

$\mathrm{dB}$. In the above equations, $\left(R_{0}\right)$ is electrical resistance, $\left(C_{0}\right)$ is capacitance volume, $(N)$ is transduction coefficient, $\left(R_{\mathrm{e}}\right)$ is resistance terms (in the mass and spring system), $(V)$ is the applied electric voltage, and $(F)$ is the generated force. ${ }^{10}$ Other used parameters are introduced in Table 3. ${ }^{16}$

Now, the results obtained from the three conditions are analysed and interpreted on the basis of the above equations and functions.

\subsection{Condition 1}

In Eq. (15), by increasing the ring's radius, its stiffness increases in the thickness direction. On the other hand, by increasing the radius, its mass would also be greater. Therefore, in the equation of mechanical impedance, the mass and stiffness terms are increasing. The third term of Eq. (20) also increases in light of enlargement of the cross area. As in this condition, the acoustical environment is air and the radiation impedance is very minute, and hence it is possible to neglect the term resistance in comparison to the term's stiffness and mass in the Eq. (20). However, in Eq. (19), increasing the external radius leads to an increase in the cross area, and $N$ becomes larger. In Eq. (18), $C_{0}$ also increases with an increase in the cross area. Considering power 2 on $N$, it could be stated that this term $(N)$ has a high effect on the final result. Referring to Eq. (16), it becomes obvious that by increasing $N$ and $C_{0}$, electrical impedance lowers. (Effect of increase in and is dominant over increase in stiffness and mass). On the other hand, it is obvious that by increasing the radius stiffness and mass, according to Eq. (22), the frequency resonance in which minimum impedance exists has also decreased.

It is noteworthy that by referring to the available relations for the matrix model, it is possible to observe the effect of change in the external radius or any other geometrical parameters on the electrical impedance of a piezoceramic ring. Clearly, by increasing the external radius, the radius resonance mode begins to reduce, such that by a large increase in the external radius, the next radius modes could also be considered within a limited frequency range (as shown by the yellow lines of the above figure).

\subsection{Condition 2}

First, consider the equation related to mechanical impedance. By increasing the thickness of the ring, its stiffness becomes larger. On the other hand, increasing the thickness causes the stiffness to reduce in the direction of thickness. That is to say, by having fixed values for the internal and external radii and by increasing the thickness, the mass has increased and the stiffness has decreased. 
Therefore, the change in the mechanical impedance depends on the numerical values of mass and stiffness. By increasing thickness, $N$ and $C_{0}$ will also decrease according to Eqs. (18) and (19). Considering the severe effect of $C_{0}$ and particularly $N$ on the electrical impedance, the input electrical impedance increases (see Eq. (16)). In order to survey the change in frequency resonance related to the radius mode, one has to pay attention to the stiffness along the radius. Since the increase in stiffness along the radius and the increase in mass are both proportional to the increase in the side surface of the ring, the ratio related to frequency resonance will remain almost steady. For this reason, the location of the occurrence of frequency resonance becomes noticeable. It is obvious that to calculate the frequency resonance of the thickness mode, in light of having its value expressed by Eq. (15) as length of ring thickness, the stiffness has a large reduction along the thickness, and thus resonance frequency would be reduced in response to an increase in thickness. All this is despite the selected frequency range; no thickness mode around would be observed.

\subsection{Condition 3}

It is observed that a change in the acoustical environment has no effect on resonance frequencies. It only caused the impedance diagram to be damped. Changing the acoustical environment from air to water with a wave propagated in it causes the value of being mass generated by effect of acoustical environment $M_{\mathrm{r}}$ to increase. However, the main effect of this change in the acoustical environment shows itself in the severe increase in the value of radiating impedance $R_{\mathrm{e}}$. The role of $R_{\mathrm{e}}$ in the system is similar to that of a damper. Although, in the environment of water, compared to air, the value of $M_{\mathrm{r}}$ has increased some. However, it is possible to neglect the changes in stiffness and mass. This means that in the system shown in Fig. 11, the mass and stiffness have remained almost constant, and the damping term has increased. Therefore, according to Eq. (22), the resonance frequencies of the system would not become changed. On the other hand, it is obvious in a mass and spring system that by an increase in the damping term, the vibration of system moves toward being damped. This means that resonance modes in the impedance diagram begin to disappear. It is for this reason that despite frequency resonance remaining constant, it appears that input electrical impedance has become damped and resonance frequencies responsive to the minimum points in the diagram are no longer sharp and are not vividly noticed.

\section{CONCLUSIONS}

Modelling of different elements of electroacoustic transducers is an introduction for the modelling of the transducers. Undoubtedly, the most important part, one that has the greatest effect on response of a transducer, is the piezoceramic ring used in it. Precise recognition of the effect of geometrical parameters of a ring helps to reach a desired frequency response based on making necessary changes in its dimensions and sizes. In most studies conducted in the past, the main emphasis has been on the analytical models. However, by considering the limitations of these models, it was attempted herein to extend the modelling to the FEM, as well. In the present paper, attempts were made to use the matrix method and the FEM simultaneously. Differences between them were studied, and the limitations and advantages of each method were analysed. Based on the presented results herein, the following findings could be reviewed as the most noticeable points of this study:

- Comparisons between the results obtained from both the matrix method and the finite element method for modelling a known ring revealed that results are very similar. Also, considering the available experimental results for the same ring and comparing both methods to experimental results indicated that both models are capable of simulating frequency behaviour of a piezoceramic ring.

- By studying the effects of geometric parameters and the surrounding environment on the frequency response of a ring, it could be stated that geometric parameters, besides having effect on electric impedance, also cause noticeable changes in the resonance frequency of a ring. This is where the surrounding environment of the ring only causes changes in the intensity of the electric impedance of the ring.

- Based on the results from both the simulation methods, by increasing the external radius of the circular ring, the resonance frequency related to the radius mode decreases. Through quantitative observation, it could be stated that by doubling the size of the external radius, the resonance frequency decreases around $30 \%$. The reduction of electric impedance responsive to this reducing radius is around $14 \mathrm{~dB}$.

- Based on the obtained results, thickness change in piezoceramic ring has no effect on resonance frequency related to radial mode. Nevertheless, these changes cause the resonance frequency of the thickness mode to be changed. Therefore, the greatest effect of change in the radius is in the resonance frequency of the radial mode, and the greatest effect of the changes in thickness is in the resonance frequency of the thickness mode. Also, by increasing thickness, the level of electrical impedance for a certain frequency would be increased.

- Based on the obtained results, changes in the acoustic environment have little effect on the location of resonance frequency. This is where change in the surrounding environment of a ring in the thickness direction from air to water has caused the shape of the impedance diagram to become damped considerably. In other words, maximum and minimum points could no longer be observed as sharply as they were before. That is to say, some resonance modes begin to disappear.

\section{REFERENCES}

1 Hodges, R. P. Underwater Acoustics, Analysis, Design and Performance of Sonar, John Wiley \& Sons, Ltd, (2010). http://dx.doi.org/10.1002/9780470665244. 
2 Etter, P. C. Underwater Acoustic Modelling and Simulation, Spon Press, (2003), $3^{\text {rd }}$ ed. http://dx.doi.org/10.4324/9780203417652.

3 Waite, A. D. Sonar for Practicing Engineering, John Wiley \& Sons, Ltd, (1988), $3^{\text {rd }}$ ed.

4 Jensen, F. B., Kuperman, W. A., Porter, M. B., and Schmidt, H. Computational Ocean Acoustics, Springer, (2011), $2^{\text {nd }}$ ed. http://dx.doi.org/10.1007/978-1-4419-8678-8.

5 Everst, F. A. The Master Handbook of Acoustics, McGraw-Hill, (2001), $4^{\text {th }}$ ed., Chapter 1. http://dx.doi.org/10.1036/0071399747.

6 Xavier, L. An Introduction to Underwater Acoustics, Principles and Applications, Springer, (2010). http://dx.doi.org/10.1007/978-3-642-13835-5.

7 Wilson, J. S. Sensor Technology Handbook, Elsevier, Amsterdam, (2005). http://dx.doi.org/10.1016/b978075067729-5/50040-9.

8 Ricón, Y. G.-U. and de Espinosa Freijo, F. M. Piezoelectric modelling using a time domain finite element program, Journal of the European Ceramic Society, 27, 4153-4157, (2007). http://dx.doi.org/10.1016/j.jeurceramsoc.2007.02.127.

9 Abdel Fattah, A. M., Ali, M. G. S., Elsyed, N. Z., and Gharieb, A. A. Piezocermaic materials for ultrasonic probes, Egyptian Journal of Solids, 28, (2005).

${ }^{10}$ Çepni, K. A Methodology for Designing Tonpilz-Type Transducers, MSc thesis, Middle East Technical University, (2011).

11 Sherman, C. H. and Butler, J. L. Transducers and Arrays for Underwater Sound, Springer, (2007). http://dx.doi.org/10.1007/978-0-387-33139-3.

12 Jovanović, I., Mančić, D., Paunović, V., Radmanović, M., and Mitić, V. V. Metal rings and discs Matlab/Simulink 3D model for ultrasonic sandwich transducer design, Science of Sintering, 44, 287-298, (2012). http://dx.doi.org/10.2298/sos1203287j.
13 Mančić, D., Radmanović, M., Petrušić, Z., and Stančić, G. Influence of ultrasonic transducer acoustic impedances and dimensions on its input electrical impedance, Working and Living Environmental Protection, 5, 59-72, (2008).

14 Kocbach, J. Finite Element Modelling of Ultrasonic Piezoelectric Transducers: Influence of Geometry and Material Parameters on Vibration, Response Functions and Radiated Field, PhD dissertation, University of Bergen, (2000).

15 Bayliss, C. Application and Development of Finite Element Techniques for Transducers Design and Analysis, PhD dissertation, University of Birmingham, (1998).

16 Radmanović, M. D. and Mančić, D. D. Designing and Modelling of the Power Ultrasonic Transducers, M.P. Interconsulting, (2004).

17 Nygren, M. W. Finite Element Modelling of Piezoelectric Ultrasonic Transducers, MSc thesis, Norwegian University of Science and Technology, (2011).

18 Chung, G.-S. and Phan, D.-T. Finite element modelling of surface acoustic waves in piezoelectric thin films, Journal of the Korean Physical Society, 57, 446-450, (2010). http://dx.doi.org/10.3938/jkps.57.446.

19 Nguyen, K. K. Design and Comparison of Single Crystal and Ceramic Tonpilz Transducers, MSc thesis, University of Texas at Austin, (2010).

20 Leping, F. and Ramanathan, S. K. On application of radiation loss factor in the prediction of sound transmission loss of a honeycomb panel, International Journal of Acoustics and Vibration, 17, 47-51, (2011).

21 Pantea, C., Osterhoudt, C. F., and Sinha, D. N. Determination of acoustical nonlinear parameter $\beta$ of water using the finite amplitude method, Ultrasonics, 53, 1012-1019, (2013). http://dx.doi.org/10.1016/j.ultras.2013.01.008. 